\title{
Universal Static and Dynamic Properties of the Structural Transition in $\mathrm{Pb}\left(\mathrm{Zn}_{1 / 3} \mathrm{Nb}_{2 / 3}\right) \mathrm{O}_{3}$
}

\author{
C. Stock, ${ }^{1}$ R. J. Birgeneau, ${ }^{1}$ S. Wakimoto, ${ }^{1}$ J. S. Gardner,${ }^{2}$ W. Chen,${ }^{3}$ Z. -G. Ye ${ }^{3}$ and G. Shirane ${ }^{4}$ \\ ${ }^{1}$ Department of Physics, University of Toronto, Ontario, Canada M5S 1A7 \\ ${ }^{2}$ National Research Council, Chalk River, Ontario, Canada, K0J 1J0 \\ ${ }^{3}$ Department of Chemistry, Simon Fraser University, Burnaby, British Columbia, Canada V5A $1 S 6$ \\ ${ }^{4}$ Physics Department, Brookhaven National Laboratory, Upton, New York 11973
}

(Dated: June 24, 2018)

\begin{abstract}
The relaxors $\mathrm{Pb}\left(\mathrm{Zn}_{1 / 3} \mathrm{Nb}_{2 / 3}\right) \mathrm{O}_{3}(\mathrm{PZN})$ and $\mathrm{Pb}\left(\mathrm{Mg}_{1 / 3} \mathrm{Nb}_{2 / 3}\right) \mathrm{O}_{3}$ (PMN) have very similar properties based on the dielectric response around the critical temperature $T_{c}$ (defined by the structural transition under the application of an electric field). It has been widely believed that these materials are quite different below $T_{c}$ with the unit cell of PMN remaining cubic while in PZN the low temperature unit cell is rhombohedral in shape. However, this has been clarified by recent highenergy x-ray studies which have shown that PZN is rhombohedral only in the skin while the shape of the unit cell in the bulk is nearly cubic. In this study we have performed both neutron elastic and inelastic scattering to show that the temperature dependence of both the diffuse and phonon scattering in PZN and PMN is very similar. Both compounds show a nearly identical recovery of the soft optic mode and a broadening of the acoustic mode below $T_{c}$. The diffuse scattering in PZN is suggestive of an onset at the high temperature Burns temperature similar to that in PMN. In contrast to PMN, we observe a broadening of the Bragg peaks in both the longitudinal and transverse directions below $T_{c}$. We reconcile this additional broadening, not observed in PMN, in terms of structural inhomogeneity in PZN. Based on the strong similarities between PMN and PZN, we suggest that both materials belong to the same universality class and discuss the relaxor transition in terms of the three-dimensional Heisenberg model with cubic anisotropy in a random field.
\end{abstract}

PACS numbers:

\section{INTRODUCTION}

The relaxor materials of the chemical form $\mathrm{PbBO}_{3}$ have generated much interest recently due to their large piezoelectric constants which are an order of magnitude larger than those of conventional ferroelectrics $\frac{1.2}{2}$ These materials are characterized by quenched disorder on the B site and display a diffuse phase transition with a broad and frequency dependent dielectric response peaked at the temperature $T=T_{\max }$ (reproduced in the upper panel of Fig. (1) $?^{3}$ Early studies of the refractive index showed that regions of local ferroelectric order are formed in a paraelectric background at a temperature $T=T_{d}$ (denoted as the Burns temperature) $\underline{\underline{\underline{4}}}$ The nature of this diffuse transition and the low temperature ground state has been the focus of many recent studies. Most recently, much attention has centered on the material $\mathrm{Pb}\left(\mathrm{Mg}_{1 / 3} \mathrm{Nb}_{2 / 3}\right) \mathrm{O}_{3}(\mathrm{PMN})$ with a relatively accessible Burns temperature of $T_{d} \sim 620 \mathrm{~K}$.

High temperature neutron inelastic scattering was originally investigated by Naberezhnov et $a l^{\underline{\underline{5}}}$ and was followed by Gehring et al $\underline{\underline{6}}^{\underline{6}}$ who clearly showed the presence of a soft ferroelectric mode in PMN which becomes overdamped below $T_{d} \stackrel{7}{\underline{7}}$ Diffuse scattering studied by Hirota et al s $^{8}$ showed that the growth of diffuse scattering could be well described by the formation of phase-shifted polar nanoregions in which the atomic displacement was described by two components - a center of mass conserving component and another non-conserving component associated with a uniform phase shift. The con- nection between the diffuse and phonon scattering was later established through a detailed phonon study ${ }^{9}{ }^{9}$ which showed the soft optic and the transverse acoustic modes to be strongly coupled. This coupling provides a natural explanation for the presence of phase-shifted polar nanoregions as the center of mass conserving atomic shift can be attributed to the condensation of the soft optic mode while the non-conserving component corresponds to a softening of the acoustic mode.

Unpoled PMN shows essentially no structural change through $T_{c}=213 \mathrm{~K}$ with only a small transverse broadening in the Bragg peaks $\frac{9}{9}$ The transition temperature $T_{c}$ in PMN is defined by the structural transition after poling the sample under an electric field $\stackrel{10}{\underline{10}}$ Despite no structural phase transition the soft optic mode in unpoled PMN was found to recover below $T_{c}$ and the energy squared was observed to increase linearly with decreasing temperature ${ }^{11}$ This is extremely surprising as such a recovery has previously been thought to be associated with a well defined structural transition ${ }^{12}$ The recovery of the soft mode suggests the presence of a ferroelectric distortion in PMN, despite the absence of any change in the average unit cell shape.

Until very recently, $\mathrm{Pb}\left(\mathrm{Zn}_{1 / 3} \mathrm{Nb}_{2 / 3}\right) \mathrm{O}_{3}(\mathrm{PZN})$ and PMN were thought to be very different with PMN remaining cubic at all temperatures and PZN undergoing a structural phase transition to a rhombohedral phase $\frac{13}{3}$ at a very well defined critical temperature $T_{c}=410 \mathrm{~K}$. The Bragg scattering around $T_{c}$ in both these compounds displays contrasting behavior as illustrated in the lower panel of Fig. 1 which shows a large increase in the neu- 


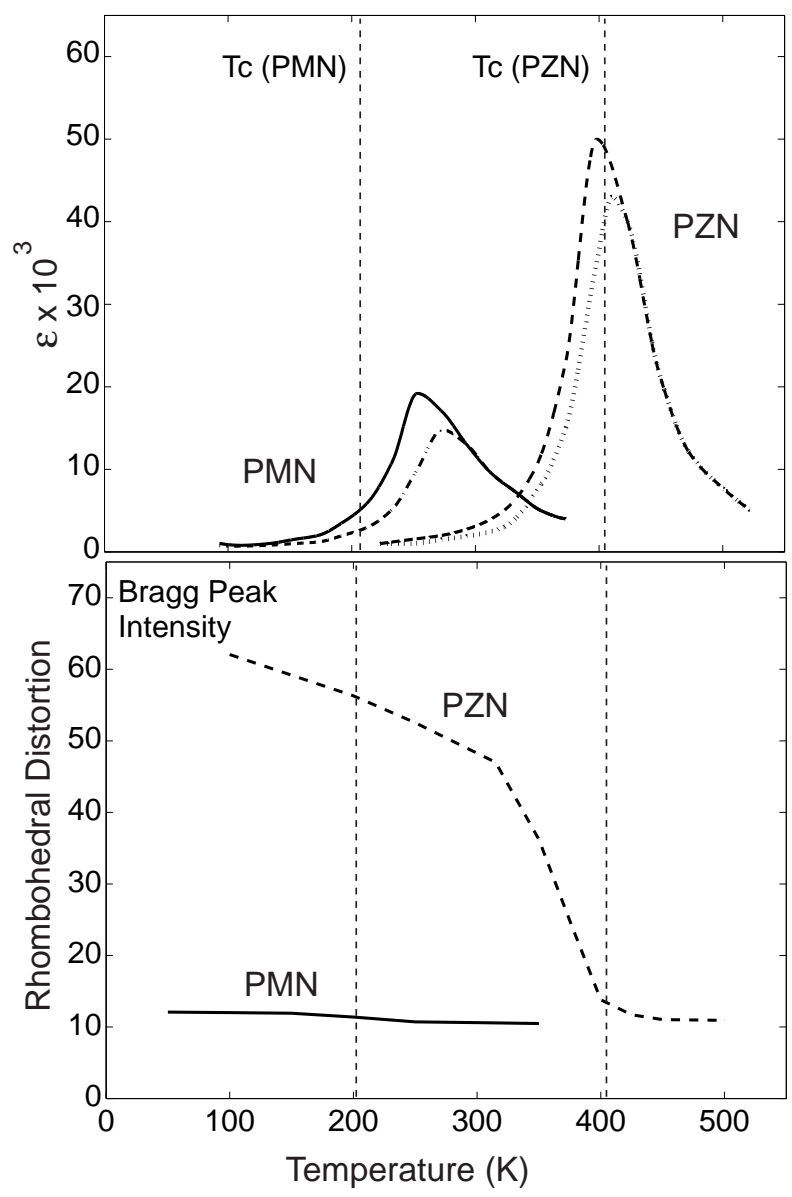

FIG. 1: Schematic figure showing the temperature dependence of the dielectric constant (data taken from Ye et al.) and the Bragg peak intensity for both PMN (220) and PZN (300) which characterize rhombohedral distortion. The upper figure shows the dielectric constant for $100 \mathrm{~Hz}$ (peaked at lower temperatures) and $1 \mathrm{MHz}$ (peaked at higher temperatures). The dotted lines indicate the critical temperature measured under the application of an electric field.

tron Bragg peak intensity for PZN at $T_{c}$ but no change in PMN over a broad range in temperature. The structural transition in PZN has been reexamined in detail by a recent diffraction study using $\sim 9 \mathrm{keV}$ x-rays 14 . Studies of the diffuse scattering in PZN by La-Orauttapong et al. showed that the diffuse scattering started near $T_{c}$, well below $T_{d}$ where the onset of diffuse scattering occurs in PMN, highlighting another difference between PMN and $\mathrm{PZN}, 15$

The notion that the unit cell in PZN undergoes a transition from a cubic to rhombohedral in shape at a well defined transition temperature $T_{c}$ changed with a recent high-energy and penetrating x-ray study by $\mathrm{Xu}$ et al 16 and with a neutron diffraction study on $\mathrm{PbTiO}_{3}(\mathrm{PT})$

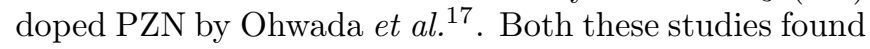
that the low temperature structural phase was not rhombohedral but rather was characterized by a cubic unit cell, denoted as phase $\mathrm{X}$. The $67 \mathrm{keV}$ high energy $\mathrm{x}-$ ray study revealed phase $\mathrm{X}$ to be characterized by resolution limited Bragg peaks suggesting a well ordered low-temperature structure. The connection to the PMN system was made recently by a high resolution neutron diffraction study on $10 \% \mathrm{PT}$ doped $\mathrm{PMN}^{18}$ which found the low temperature ground state to also be characterized by a cubic shaped unit cell with nearly resolution limited Bragg peaks. These recent studies on PZN and PT doped PMN show that PZN and PMN may not be as different as once thought. These results necessitate a more detailed study of the dynamical and static properties of pure PZN and a comparison between PZN and PMN.

To investigate the analogy between PMN and PZN we have conducted a detailed study of the structural and dynamical properties of PZN around the critical temperature $T_{c}$ using neutron inelastic and elastic scattering. We will define the similarities between PMN and PZN through both the inelastic scattering from the phonons and the elastic diffuse scattering. A key difference between these relaxor systems illustrated by the Bragg scattering will be discussed and reconciled in terms of structural inhomogeneity in PZN. The strong similarity between these two materials suggests they belong to the same universality class. We propose that the relaxor transitions can be understood in terms of the threedimensional Heisenberg model with cubic anisotropy in the presence of a random field. This model is very attractive as it unifies the many temperature scales measured by various experimental probes.

\section{EXPERIMENTAL DETAILS}

Neutron scattering experiments were performed at the NRU reactor, Chalk River Laboratories. Elastic scattering studies of the diffuse scattering and the Bragg peaks were conducted on the N5 spectrometer by holding the incident and final energies fixed at $14.8 \mathrm{meV}$. Diffuse scattering results were obtained using a flat Graphite (002) monochromator and analyzer. Horizontal collimation was set by Soller slits from the reactor to detector at $77^{\prime}-33^{\prime}-S-35^{\prime}-60^{\prime}$ (where $S$ denotes the sample) and a Graphite filter was used on the scattered side to remove higher order neutrons. For elastic studies of the Bragg peaks we have matched the $d$-spacings of the sample to that of the (220) reflection of a Germanium analyzer with a small mosaic of $\sim 3^{\prime}$. By matching the $d$-spacings of the sample and analyzer crystal we have obtained good resolution allowing us to study the small broadening of the Bragg peaks below $T_{c}$. For elastic measurements of the Bragg peaks we have also set the horizontal collimation to $77^{\prime}-6^{\prime}-S-12^{\prime}-60^{\prime}$.

Inelastic measurements studying both the transverse acoustic and optic modes were conducted on the C5 spectrometer by fixing the final energy to $14.5 \mathrm{meV}$ and varying the incident energy. A variable focusing graphite (002) monochromator and a flat graphite analyzer were 
used with the horizontal collimation set at $33^{\prime}-29^{\prime}-S-48^{\prime}-$ $72^{\prime}$ to study the acoustic mode and $33^{\prime}-48^{\prime}-S-51^{\prime}-72^{\prime}$ to study the optic mode. A graphite filter was also used on the scattered side to filter out higher order neutrons and a cold sapphire filter was used before the monochromator to reduce the fast neutron background.

Single crystals of PZN were grown by spontaneous nucleation from high temperature solution using $\mathrm{PbO}$ as flux, based on the technique previously described ${ }^{19} \mathrm{~A}$ single crystal with a volume of $0.2 \mathrm{cc}$ and a weight of 1.8 $\mathrm{g}$ was selected for the experiments. It was oriented with the largest face parallel to the (100) cubic plane. The sample of PZN was mounted, using tantalum wire, to a boron-nitride post with a copper insert to ensure good thermal contact. The sample was mounted in an orange cryofurnace such that reflections of the form (HK0) lay in the scattering plane. The room temperature lattice constant was measured to be $4.04 \AA$ and therefore 1 reciprocal lattice unit (r.l.u.) corresponds to $2 \pi / a=1.55$ $\AA^{-1}$. Because PZN decomposes at very high temperatures we have kept our high temperature measurements below $550 \mathrm{~K}$ to avoid any possible damage. Unfortunately, this means that the studies above or around the Burns temperature $T_{d}$, which exceeds $670 \mathrm{~K}$, are not possible in PZN.

\section{BRAGG SCATTERING}

To investigate the Bragg peak profile as a function of temperature around $T_{c}$ we have used a Germanium analyzer with a small mosaic spread of $\sim 3^{\prime}$. Based on low-energy x-ray results showing a transition from cubic to rhombohedral we conducted scans around the (110) position. If a rhombohedral phase exists at low temperatures the Bragg peak should split along the longitudinal [110] direction only. Instead of such a distortion we observe a broadening of the (110) Bragg peak along both the longitudinal [110] and transverse [1 10 ] directions (Fig. 21). This is in contrast to the behaviour in PMN which shows only transverse broadening below $T_{c}$ (Ref. 9). We have also investigated the temperature dependence of the (200) Bragg peak which is found to also display both transverse and longitudinal broadening below $T_{c}$ (Fig. 3). As mentioned in the next section, we find no observable temperature dependent diffuse scattering around the (200) position. This means that the broadening of the Bragg peaks shown in Fig. 3] cannot be associated with the diffuse component.

These results contrast with the results of both lowenergy and high energy x-ray measurements. The $\sim 9$ keV x-ray study by Lebon et al. showed the ground state of PZN at low temperatures to have a rhombohedral unit cell. The high-energy $67 \mathrm{keV}$ x-ray results by $\mathrm{Xu}$ et al. found the unit cell to be nearly cubic and to be very well ordered characterized by resolution limited Bragg peaks. $\mathrm{Xu}$ et al. also confirmed the result of Lebon et al. by showing that a splitting of the (111) Bragg peak charac-

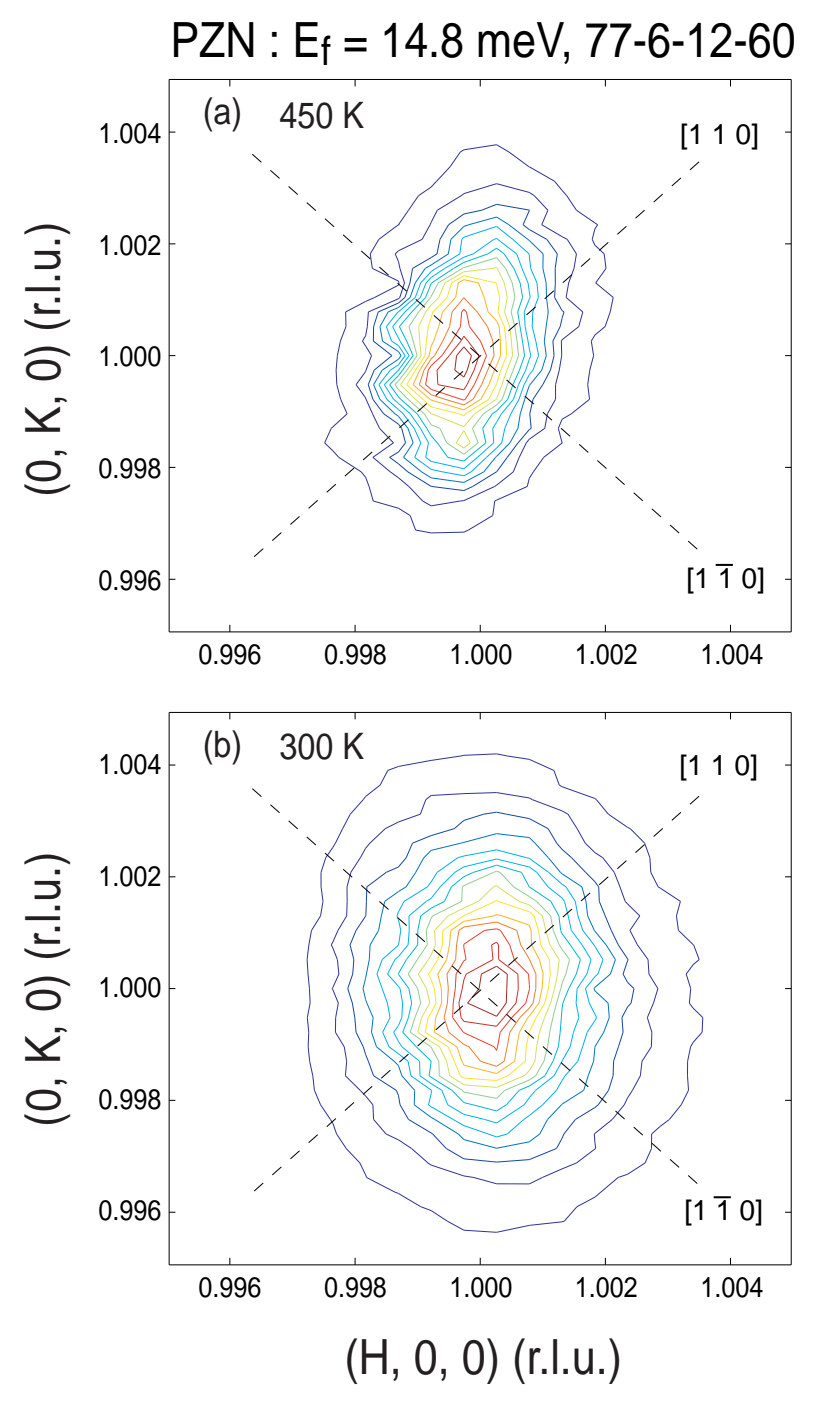

FIG. 2: Contour plots of the (110) reflection as scanned in the $(H K O)$ plane above $(a)$ and below $(b)$ the critical temperature $T_{c}$. These data were taken using a Germanium analyzer with a small mosaic spread. Dashed lines indicate longitudinal [110] and transverse [1̄̄0] directions.

teristic of a rhombohedral distortion existed when a lower incident beam energy of $32 \mathrm{keV}$ was used. The contrasting results presented by $\mathrm{x}$-ray scattering with various incident energies can be reconciled based on the relative penetration depths. As noted by $\mathrm{Xu}$ et al., the lower energy $\mathrm{x}$-rays in the range $10-32 \mathrm{keV}$ have a penetration depth in PZN of only $\sim 10-60 \mu \mathrm{m}$ whereas x-rays with an incident energy of $67 \mathrm{keV}$ have a penetration depth of $\sim 400 \mu \mathrm{m}$ which should give a more accurate picture of the bulk properties. Therefore, in PZN rhombohedral order is formed in the skin of the sample whereas the bulk has a well ordered pseudocubic structure. This new bulk ground state has been referred to as Phase-X.

Our neutron results showing both transverse and longitudinal broadening of the Bragg peaks are consistent with the picture obtained from x-rays. Since neutrons 

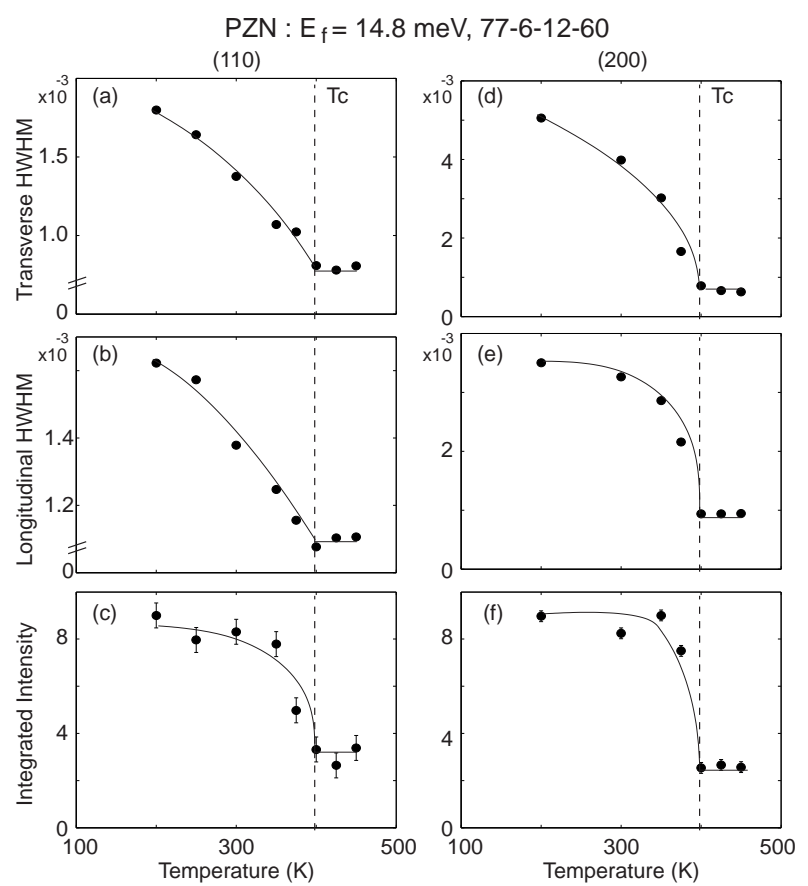

FIG. 3: Plots of the transverse, longitudinal widths, and the integrated intensities as a function of temperature. The widths were obtained from Lorentzian fits to the observed scattering. In all cases there is clear anomaly at the critical temperature $T_{c}$. The solid lines are guides to the eyes.

probe the entire sample volume the observed Bragg peak profile is a combination of both the bulk and the nearsurface region. The presence of both transverse and longitudinal broadening is consistent with finite size effects resulting from domains. Thus, based on the x-ray results, the broadening along both directions is very likely the result of structural inhomogeneity in the bulk sample. The size of these domains can be estimated from the half-width (estimated from a Lorentzian profile) ${ }^{20}$ of the Bragg peaks at low temperatures to be $\sim 200-400 \AA$. This estimate is consistent with the value of $700 \AA$ estimated by $\mathrm{Xu}$ et al. based on the low energy x-ray results.

The existence of finite size effects resulting from structural inhomogeneity is also consistent with the temperature dependence of the integrated intensity displayed in Fig. 3 for both the (110) and (200) Bragg peaks. Both Bragg peaks show a large increase in the integrated intensity at $T_{c}$ characteristic of a release of extinction at low temperatures. Such an effect is the result of a broadening of the overall mosaic spread of the crystal allowing better penetration of the neutron beam into and out of the crystal. Detailed analysis of extinction and its dependence on the domain size is discussed elsewhere ${ }^{21}$

Thus, our results are consistent with the high-energy $\mathrm{x}$-ray results showing the absence of rhombohedral order at low temperatures. We do observe a longitudinal line broadening indicating structural inhomogeneity. In the sense that no rhombohedral order is formed in the bulk

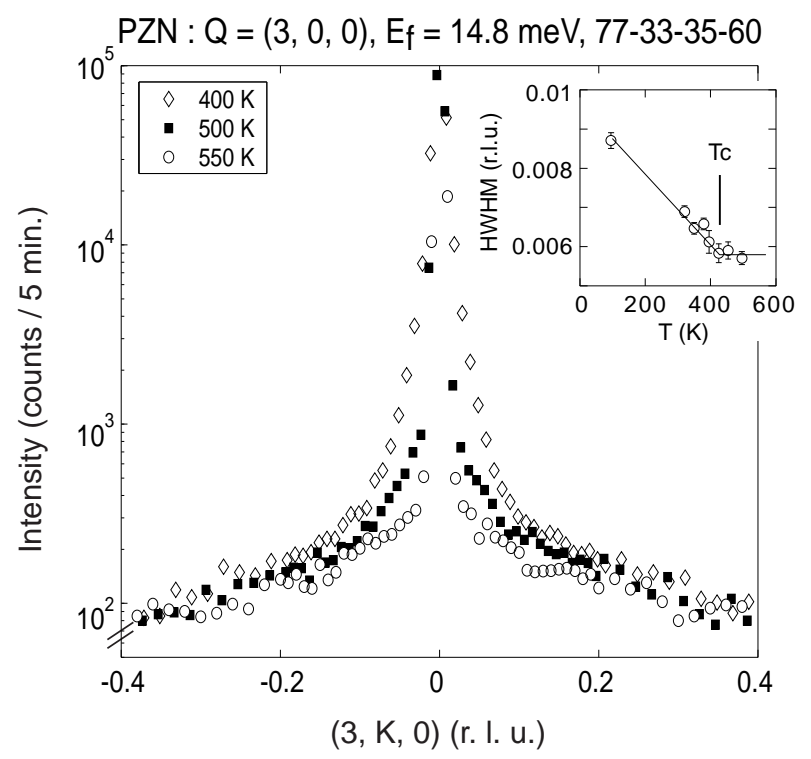

FIG. 4: Diffuse scattering around the (300) position at several temperatures. The diffuse scattering is clearly present well above the critical temperature $T_{c}=410 \mathrm{~K}$ and suggestive that diffuse scattering starts at the Burns temperature $T_{d}$. The inset shows the unconvoluted HWHM of the (300) Bragg peak as a function of temperature.

at low temperatures, the structural properties of PZN are similar to those of PMN.

\section{DIFFUSE SCATTERING}

The absence of a clear rhombohedral distortion at low temperatures necessitates a revaluation of the diffuse scattering. Previous diffuse scattering results by D. La-Orauttapong et al $!^{15}$ have indicated that the temperature dependent diffuse scattering has an onset near $T_{c} \sim 410 \mathrm{~K}$. This result contrasts with that in PMN where the diffuse scattering starts at the much higher temperature of $T_{d}$ where polar nanoregions are formed.

We have studied the diffuse scattering and its temperature dependence around the (300) reflection where the diffuse scattering is strong. We have found that the lineshape of the diffuse scattering at low temperatures is qualitatively similar to that of PMN studied in detail by You et $a l^{22}$ These authors have found that the diffuse intensity in PMN is elongated along both the [110] and [11̄0] directions at the (300) position. Figure 4 shows the temperature variation of the (300) diffuse profile indicating the presence of diffuse scattering even above $500 \mathrm{~K}$. The presence of a diffuse component above $T_{c}$ is clearly demonstrated in Fig. $[5$ which shows the temperature dependence of the diffuse intensity at different $q$-positions with a constant background indicated by a dashed line. It is again clear that diffuse scattering is still present at $550 \mathrm{~K}$. These results indicate that diffuse scattering starts well above $T_{c}$ and is suggestive of an onset at the 


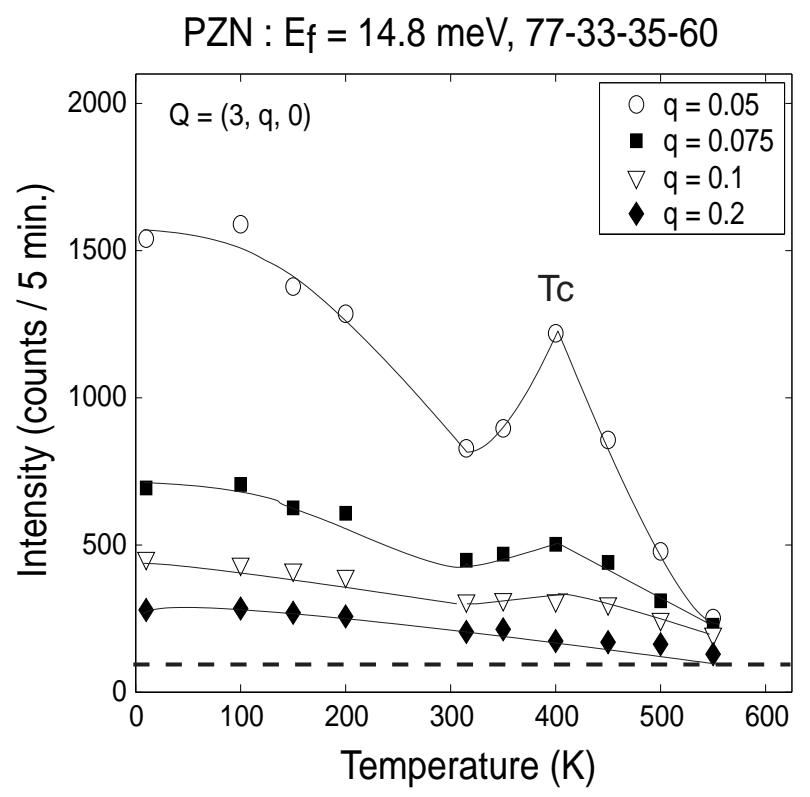

FIG. 5: Measured intensity at (3, q, 0) as a function of temperature for various values of $q$. Large values of $q$ show a gradual and continuous growth with decreasing temperature of the diffuse scattering through $T_{c}$ while smaller values of q show evidence of critical scattering. The horizontal dotted line indicates the constant background.

Burns temperature $T_{d}$ similar to the case in PMN. As also displayed in Fig. 目 this continuous growth of the diffuse scattering is in contrast to the transverse HWHM of the Bragg peak which shows a sharp anomaly at $T_{c}$. The gradual growth of the diffuse intensity starting at temperatures in excess of $500 \mathrm{~K}$ differs with the suggestion that the diffuse scattering starts at $\sim 450 \mathrm{~K}^{15}$ and is consistent with previous diffuse work on PMN which clearly showed the onset of a diffuse component at the Burns temperature .5 .8

Another important characteristic of diffuse scattering in PZN is shown in Fig. [5 For large values of $q$ the diffuse scattering grows continuously through $T_{c}$ but at smaller values of $q$ a clear peak is observed in the measured intensity suggesting the presence of critical scattering. Even though this is consistent with the diffuse scattering in $\mathrm{PMN}^{\mathrm{P} T}{ }^{23}$ these results differ from the current understanding of diffuse scattering in pure PMN which shows a continuous change through $T_{c}{ }^{24}$ Therefore the diffuse scattering around $T_{c}$ could be interpreted as consisting of scattering from the polar nano-regions formed at $T_{d}$ and critical scattering from some component of the sample undergoing a structural phase transition at $T_{c}$ (see Fig (1). Based on our analysis of the Bragg scattering this extra component of the sample which undergoes a structural transition is located in the near-surface region and is the same component studied by Lebon et al. using low energy x-rays.

We believe that the structural inhomogeneity is the underlying reason for the apparent discrepancy between our diffuse scattering results and those of a previous diffuse study on pure unpoled PZN by D. La-Orauttapong $\stackrel{15}{\underline{12}}$ In that study, a two phase critical scattering model was applied to the diffuse intensity around the (110) position which used a Lorentzian function to describe the diffuse scattering and a Gaussian to describe the Bragg peak. This analysis showed the onset of the diffuse Lorentzian component near $T_{c}$ and no change in the Bragg peak width. We believe that this model is oversimplified as the scattering should be the sum of the diffuse component, the Bragg peak, and the critical scattering coming from the skin of the sample which does enter a rhombohedral phase at low temperatures. Therefore we speculate that the diffuse Lorentzian component in the fits by D. La-Orauttapong et al. reflected mostly the critical component not characteristic of the bulk phase (phase-X) of the sample.

To test further the similarity with PMN we have checked for diffuse scattering at the (200) and (110) positions at temperatures above and below $T_{c}$. We have found no observable change in the scattered intensity around the (200) Bragg peak position and only a small change around the (110) Bragg peak. This implies the absence of diffuse scattering around (200) and only a weak diffuse component around (110). These results for the structure factors are predicted by the phase shifted polar nanoregion model used to describe the diffuse scattering in PMN. In this model the atomic displacements are divided into a component which conserves the center of mass and another, associated with an overall phase shift, which does not. The component associated with the center of mass conserving optic modes is determined by a linear combination of the Last and Slater modes 25 Based on a calculation similar to that conducted by Hirota et al. and assuming the same parameters for the product of the atomic displacement and the atomic mass at each lattice site as in PMN we find the calculated phase shift for PZN to be $\delta_{\text {shift }} \sim 0.56$ (normalized to the total shift of the $\mathrm{Pb}$ atom). This is quite similar to that of PMN, $\delta_{\text {shift }} \sim 0.58$. Hirota et al. found by choosing the correct weight of both Last and Slater modes and using the calculated phase shift, the intensity of the diffuse scattering at the (200) position could be made small compared to the diffuse scattering at the (110) and (300) positions. This is the same trend observed in PZN.

The absence of any observable temperature dependent diffuse component around the (200) position is also important as it illustrates that the line broadening observed in the Bragg scattering is coming from structural effects with an onset at $T_{c}$ and not from a diffuse component (see Fig. 31). This result contrasts with the previous suggestion that the apparent change in the Bragg peak widths were a result of a strong diffuse temperature dependence around $T_{c}, 15$ Clearly, the Bragg peaks show an anomalous linewidth broadening beginning at the critical temperature $T_{c}$ in PZN (see Fig. 3).

Our analysis based on the temperature dependence and structure factors link the diffuse scattering directly 
with the formation of polar nanoregions. Despite the fact that we are not able to reach the Burns temperature $T_{d} \sim 700 \mathrm{~K}$ our results suggest that the onset of the diffuse scattering starts at $T_{d}$ (where polar nanoregions are formed). Our results are strongly analogous to those in PMN where neutron scattering has shown the presence of a diffuse component at the accessible Burns temperature $T_{d} \sim 620 \mathrm{~K}$.

\section{LATTICE DYNAMICS}

In the previous sections, we have shown that PZN has similar diffuse scattering characteristics to those of PMN. Another important feature closely related to the relaxor mechanism are the properties of the transverse optic and acoustic phonon modes. These phonon modes in PMN have been well studied. Striking features in PMN appear around $T_{c}$, such as recovery of the soft optic mode below $T_{c}$ and disappearance of the acoustic phonon line broadening. In this section, we report the transverse optic and acoustic phonons in PZN measured around $T_{c}$ and compare in detail these results to those of PMN to illustrate the similarity between these two relaxor systems. Firstly, we introduce the lineshape used to analyze our phonon results.

\section{A. Lineshape: Simple Harmonic Oscillator}

Measurement of the neutron scattering intensity provides a direct measure of $\mathrm{S}(\mathbf{Q}, \omega)$, which is related to the imaginary part of the susceptibility $\chi^{\prime \prime}(\mathbf{Q}, \omega)$ by the fluctuation dissipation theorem ${ }^{26}$,

$$
S(\mathbf{q}, \omega)=\pi^{-1}[n(\omega)+1] \chi^{\prime \prime}(\mathbf{q}, \omega),
$$

where the energy transferred to the sample is defined by $\hbar \omega=E_{i}-E_{f}$ and $n(\omega)=1 /\left(e^{\hbar \omega / k_{B} T}-1\right)$ is the Bose factor. In order to obtain detailed information on the linewidth from the inelastic spectrum, a model must be convolved with the resolution function and then fit to the observed scattering. To analyze the phonon scattering as a function of temperature we have taken $\chi^{\prime \prime}$ to be described by the formula for the damped simple harmonic oscillator. We have used the equation given by the antisymmetrized linear combination of two Lorentzians,

$$
\begin{aligned}
\chi^{\prime \prime}(\mathbf{q}, \omega) & =\frac{A}{\left[\Gamma(\omega)^{2}+\left(\hbar \omega-\hbar \omega_{\circ}(\mathbf{q})\right)^{2}\right]} \\
& -\frac{A}{\left[\Gamma(\omega)^{2}+\left(\hbar \omega+\hbar \omega_{\circ}(\mathbf{q})\right)^{2}\right]},
\end{aligned}
$$

where $\Gamma(\omega)$ is the frequency dependent half-width-athalf-maximum (HWHM), $\omega_{\circ}(\mathbf{q})$ is the undamped phonon frequency, and $A$ is the amplitude. For acoustic modes,

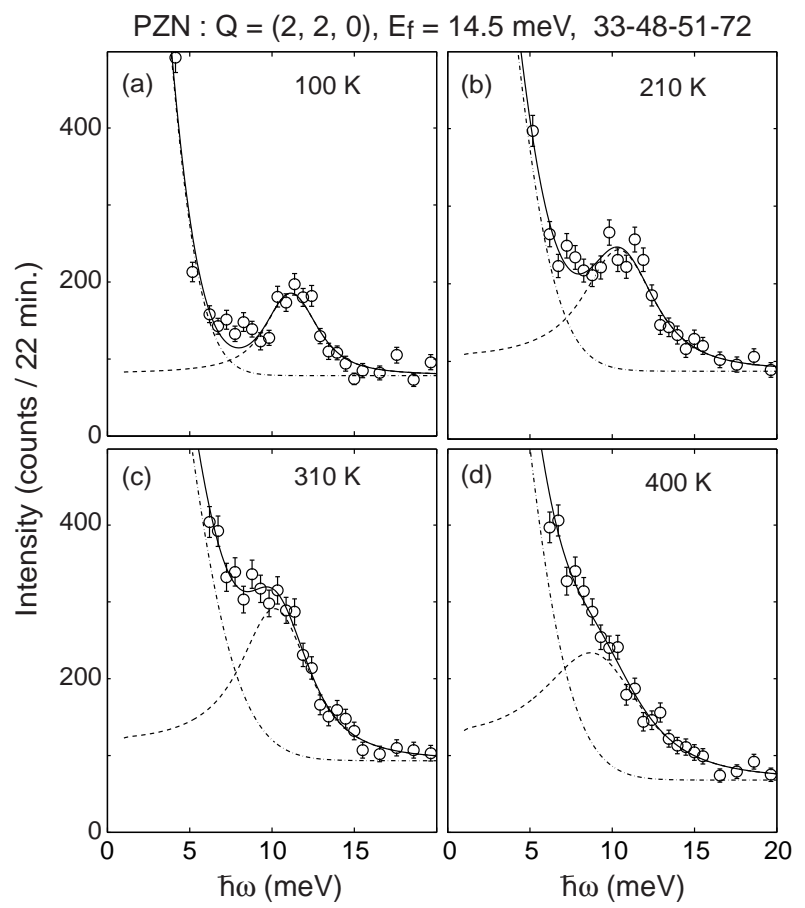

FIG. 6: Scans of the transverse optic mode at (220). The solid line is the sum of the convoluted Lorentzian and a Gaussian centered at $\omega=0$. The dashed line is the Lorentzian contribution and the dotted-dashed line is the Gaussian at zero energy transfer.

we have approximated the dispersion to be linear, $\omega_{\circ}(\mathbf{q})=c|\mathbf{q}|$, where $c$ is the phonon velocity. For optic modes, we have set the dispersion to have the form $\omega_{\circ}(\mathbf{q})^{2}=\Omega_{\circ}^{2}+(\Lambda|\mathbf{q}|)^{2}$, where $\Omega_{\circ}$ is the soft-mode energy at $q=0$ and $\Lambda$ is a constant for a given $\mathbf{q}$ direction ${ }^{27.28}$

The antisymmetrized Lorentzian lineshape obeys detailed balance ${ }^{26}$ which requires $\chi^{\prime \prime}$ to be an odd function in energy. By making several substitutions 29 the lineshape in our analysis can be shown to be equivalent to those employed in previous studies which used a frequency independent damping constant, $\Gamma_{0}, 27$ The measured intensity was fitted to Eq. 2] convolved with the instrumental resolution and a non-convoluted Gaussian function was used to describe the component at $\omega=0$. A constant was used to describe the overall background.

\section{B. Soft Optic Mode}

The soft transverse optic mode was studied by scans with fixed $\mathbf{Q}$ at the $(2,2,0)$ position. Figure 6 displays scans over a broad range of temperature below $T_{c}$. The solid lines are the results of fits to the antisymmetrized Lorentzian lineshape convoluted with the instrumental resolution. The data shows a clear softening and dampening of this mode with increasing temperature until the optic peak is essentially unresolvable around $T_{c}$ in Fig. 6 (d). We have also confirmed that the soft optic mode is 


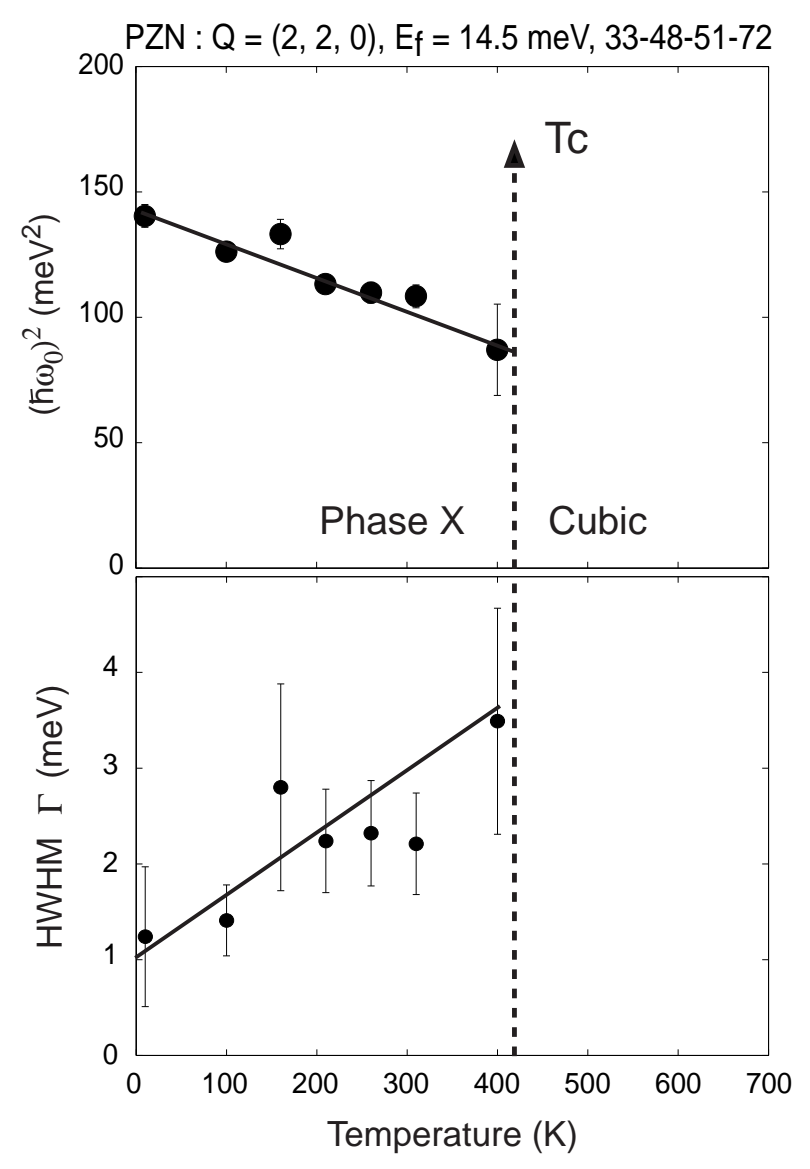

FIG. 7: Transverse optic fitted parameters as a function of temperature. The upper panel is the phonon frequency $\omega_{\circ}^{2}$ plotted as a function of temperature. The HWHM $\Gamma$ is plotted in the lower panel.

unobservable above $T_{c}$ up to at least $550 \mathrm{~K}$.

The phonon frequency squared, $\left(\hbar \omega_{\circ}\right)^{2}$, and the halfwidth are plotted in Fig. [7 The square of the phonon frequency, $\omega_{\circ}^{2}$, is related to the dielectric constant $\epsilon$ via the Lyddane-Sachs-Teller (LST) relation which states that $(1 / \epsilon) \propto\left(\hbar \omega_{\circ}\right)^{2}$. The linear relationship between the square of the phonon frequency and temperature has been established both above and below $T_{c}$ in conventional ferroelectrics 30 and is explained in the theory of Cochran ${ }^{12}$. A linear recovery of the soft optic mode has been observed in PMN at low temperatures 11 These combined results for both PMN and PZN suggests the existence of a well-defined ferroelectric distortion over the length scale characterized by the resolution $(\sim 100 \AA)$. Therefore these results point to at least a local ferroelectric polarization in the unpoled systems. Local or microdomain polarization in PZN has also been observed by Raman and optic studies 31 and it was suggested that the unpoled system had only orientational order and not translational order.

As $T_{c}$ is approached from low temperatures, the optic mode becomes gradually more damped and above the critical temperature it becomes completely overdamped

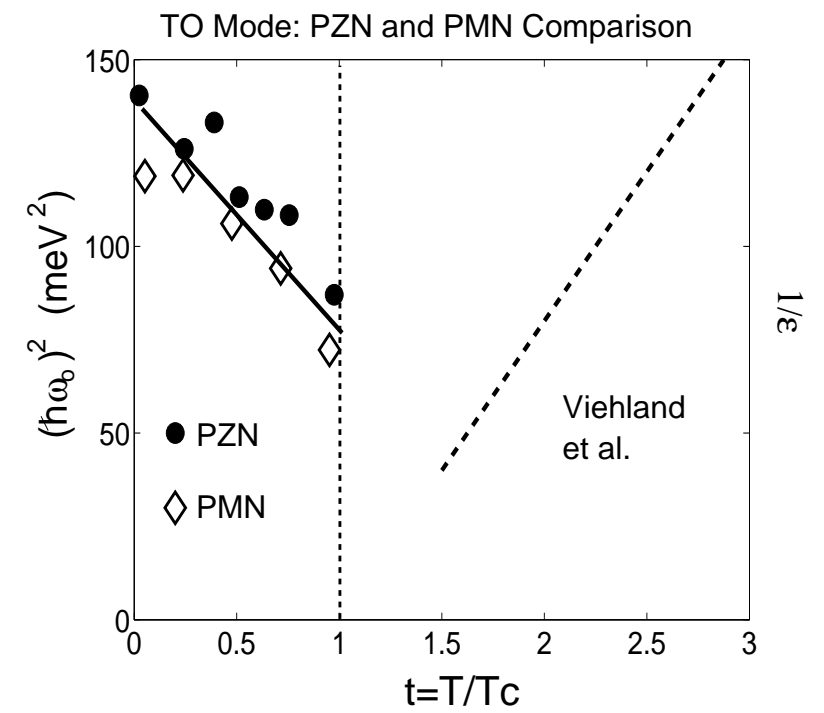

FIG. 8: The soft mode position $\left(\hbar \omega_{\circ}\right)^{2}$ as a function for reduced temperature $t=T / T_{c}$ for both PMN and PZN. The data for both materials overlaps extremely well illustrating the strong similarity between these two materials. The dashed line above $t=1$ is a schematic representation of $1 / \epsilon$ taken from Viehland et al.

and is unobservable up to $550 \mathrm{~K}$, the highest temperature studied. This is the "waterfall" region where the optic mode becomes overdamped as a result of the formation of polar nanoregions. Polar nanoregions were first suggested by Burns and Dacol $\underline{4}$ based on changes in the index of refraction below a high temperature denoted as the Burns temperature $T_{d}$. They found the data to be well described by local regions of ferroelectric order in a paraelectric background. In this temperature range, the uncorrelated polar nanoregions with random polarization prevent the propagation of the optic mode. This effect has been clearly observed in PMN where $T_{d} \sim 620$ $\mathrm{K}$ is easily accessible ${ }^{\underline{6}}$ illustrating that the temperature dependence of the soft optic mode in PZN is qualitatively similar to that in PMN. The waterfall effect and polar nanoregions are reviewed elsewhere in the context of coupled mode theory $\mathrm{I}^{\underline{7}}$ and has been verified by other groups ${ }^{32}$ in this system.

The recovery of the soft optic mode below $T_{c}$ is found to be quantitatively analogous to the recovery observed in PMN. In Fig. 8 we plot $\left(\hbar \omega_{\circ}\right)^{2}$ as a function of the reduced temperature $t=T / T_{c}$ for both PMN and PZN showing that the slope of the phonon recovery is the exactly same in both PMN and PZN. We also reproduce the high-temperature data for the dielectric constant in PMN. The data for PMN are taken from Wakimoto et $a l \stackrel{11}{\Perp}$ and the data for the high-temperature dielectric constant $(1 / \epsilon)$ is taken from Viehland et al ${ }^{33}$ Since the soft optic mode energy characterizes the degree of the ferroelectric distortion, Fig. 8 demonstrates that both PZN and PMN develop an analogous ferroelectric distortion at low temperatures. 


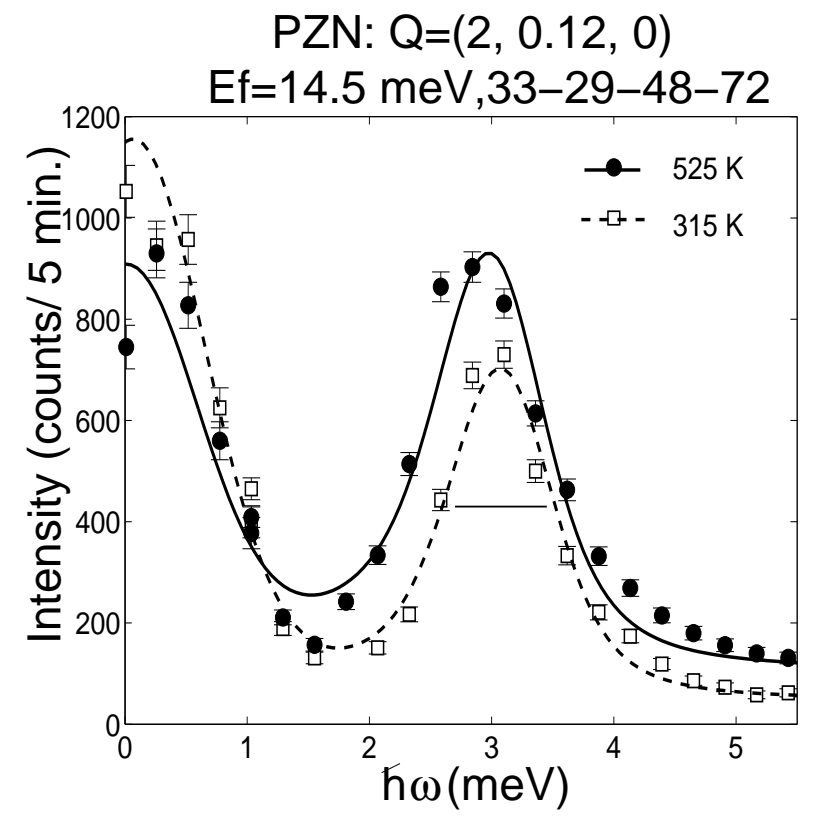

FIG. 9: Scans of the transverse acoustic (TA) mode above and below $T_{c}$ showing a small broadening above the critical temperature $\sim 400 \mathrm{~K}$. The solid and dashed lines are the results of least-squares fits to the lineshape discussed in the text convoluted with the instrument resolution. The solid bar indicates the calculated instrumental resolution FWHM.

\section{Acoustic Mode}

Studies of the transverse acoustic mode (TA) were made by conducting scans with fixed $\mathbf{Q}$ at the $(2,0.12$, $0)$ position. Representative scans above and below $T_{c}$ are displayed in Fig. 9 where the solid and dashed curves are the results of least-squares fit to the lineshape previously described convoluted with the resolution. A comparison of the results above and below $T_{c}$ shows a slight broadening at high temperatures.

The half-width is plotted as a function of temperature in Fig. 10 (a) and shows a sharp anomaly at $T_{c}$ while the integrated intensity is essentially constant over the temperature range studied. The recovery of the transverse acoustic mode for temperatures below $T_{c}$ is similar to that observed in $\mathrm{PMN}^{11}$ and $\mathrm{PT}$ doped $\mathrm{PMN}^{23}$. In both these cases the dampening of the acoustic mode appears below $T_{d}$ and is attributed to the formation of polar nanoregions. The recovery of the acoustic mode is associated with the development of local correlations between polar nanoregions resulting in local ferroelectric polarization. Since the acoustic modes are associated with center of mass motion they are less sensitive to the random field associated with the polar nanoregions and therefore should only be slightly broadened compared to the complete overdamping of the optic mode already presented.

Also, the recovery of both the optic and acoustic modes at $T_{c}$ suggests the coupling of these modes, and therefore

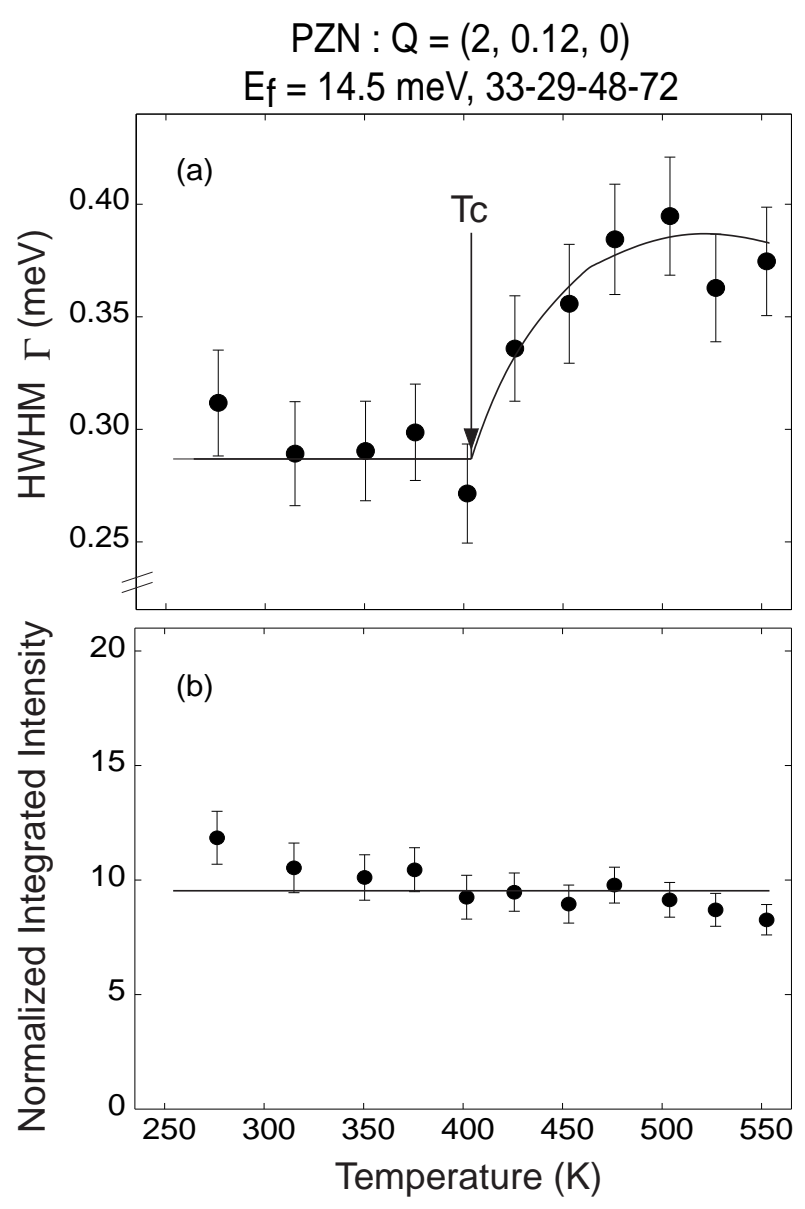

FIG. 10: Fitted parameters as a function of temperature. Figure $(a)$ shows the HWHM $\Gamma$ as a function of temperature. The integrated intensity is plotted in figure $(b)$ and has an essentially flat temperature dependence. The solid lines are guides to the eye.

the dynamics should be well described by coupled-mode theory ${ }_{28}^{28}$ This was observed to be the case in PMN where the temperature and the zone dependent lattice dynamics could be well described by coupled mode theory $\underline{\underline{\underline{9}}}$ In PMN the appearance of diffuse scattering at $T_{d}$ was interpreted in terms of the condensation of the coupled soft mode. Even though we are not able to probe temperatures near the Burns temperature $T_{d}$ the strong analogy with PMN suggests that the broadening observed in the acoustic mode starts at $T_{d}$ and is associated with the condensation of the coupled soft mode, showing another similarity between PZN and PMN.

\section{DISCUSSION AND CONCLUSIONS}

We have shown that PMN and PZN are essentially identical in terms of the temperature dependence of the diffuse scattering and the phonon scattering. The temperature dependence of the diffuse scattering is sugges- 
tive of an onset not at the critical temperature $T_{c}$, but at the much higher Burns temperature $T_{d}$ where polar nanoregions are formed. The temperature dependence of the phonon scattering has also been shown to be exactly analogous to that in PMN. The recovery of the soft optic mode in PZN is identical to the observed recovery in PMN. At temperatures above $T_{c}$, the optic mode becomes completely overdamped due to the polar nanoregions and the acoustic mode also becomes slightly damped. Both of these results are identical to those found in the PMN system.

Despite these similarities, the Bragg peaks in PZN show the onset of both transverse and longitudinal broadening at $T_{c}$ parallelled by a large growth in the integrated intensity. We have argued based on a direct comparison of our neutron results to the previous high energy $\mathrm{x}$-ray results of $\mathrm{Xu}$ et al. that this indicates the presence of structural inhomogeneity, probably in the bulk of the sample. As illustrated in Fig. 10PMN does not show any anomaly in the integrated intensity. No change is also observed in the longitudinal width and only a subtle broadening is observed in the transverse width $\frac{9}{\underline{9}}$ This suggests that the key difference between PMN and PZN is the presence of macroscopic structural disorder at low temperatures in $\mathrm{PZN}$.

A similar discrepancy in the structural properties as a function of incident x-ray energy has been observed in the doped PZN-PT system where the physical properties have been observed to vary as a function of sample thickness. This has been directly shown by Noheda et al. in the PZN-PT system and is illustrated by several experiments using both high and low energy x-rays ${ }^{34.35 .36}$ Our result, combined with those of previous x-ray studies conducted at various energies, provides further support to the growing evidence that structural inhomogeneity is a general feature of PZN and possibly to all relaxor systems. This point is further illustrated by the neutron powder diffraction work by Iwase et al. suggesting the existence of two structural phases below $T_{c}, 37.38$ We believe that previous probes sensitive to near-surface effects, like low energy x-rays with a small penetration depth and powder diffraction, showing rhombohedral order have been interpreted as a bulk result and therefore marking a clear difference between the PMN and PZN systems, whereas in fact the bulk structural phases are quite similar.

The recovery of the soft optic mode at low temperatures in both PMN and PZN points to the presence of ferroelectric polarization on a length scale of at least $\sim$ $100 \AA$ (defined by the resolution) in both systems despite the clear absence of any rhombohedral order in the bulk phase (phase $\mathrm{X}$ ). This suggests that the ferroelectric order parameter (the atomic shift or the polarization) is decoupled from the unit cell shape. This same effect was recently observed in strained $\mathrm{SrTiO}_{3}$ films ${ }^{39}$ In this system strong superlattice peaks characteristic of a structural transition were observed without any change in the c-axis lattice parameter. This was interpreted as the internal degrees of freedom (in that case the $\mathrm{TiO}_{6}$ rotations) becoming uncoupled from the overall lattice shape. In PZN and PMN a ferroelectric polarization is indicated by the recovery of the soft optic mode without the presence of a rhombohedral distortion. This fact suggests that the atomic shift is decoupled from the unit cell shape in phase $\mathrm{X}$. This picture is similar to that of Lebon et al ${ }^{31}$ who have suggested that local orientational order (microdomains) characterizes the low temperature ground state (phase-X). We note though that phase-X is structurally well ordered as characterized by the resolution limited Bragg peaks observed by $\mathrm{Xu}$ et al. using high energy x-rays.

Therefore, the characteristic feature of relaxors PZN and PMN is possibly a weak coupling between the ferroelectric order parameter and the structure as seen in the unit cell shape. In the case of conventional ferroelectric systems like $\mathrm{BaTiO}_{3}$ the coupling is strong resulting in a well defined structural transition with long-range translational and orientational order. In the case of PMN the coupling is weaker resulting in the development of local ferroelectric polarization without any change in the unit cell shape. In PZN, the coupling is still weaker; thus the bulk phase shows no change in unit cell. However the coupling is slightly larger than that of PMN, therefore resulting in a well defined change in the unit cell shape with the aid of some extra strain as found near the surface of a sample.

The strong similarity between PMN and PZN and the nature of the relaxor phase transition in both materials, as discussed above, point to a common model for the relaxor transition. Hereafter we discuss a universality class which possibly describes the relaxor transition from the viewpoint of an analogy of the polarization vector to magnetic spins. When trying to formulate a model for the relaxor transition it is important to consider that there are two key temperatures which need to be described; the Burns temperature $T_{d}$ and the lower critical temperature $T_{c}$. Also, the model needs to explain the reason for the history dependence of measured quantities around $T_{c}$. To satisfy these constraints, we propose the three-dimensional Heisenberg model with cubic anisotropy universality class in the presence of random fields. Variants of this model have been considered previously although our particular formulation and analysis appear to be unique. ${ }^{40.41 .42}$ The mapping is as follows: the Heisenberg spin corresponds to the local ferroelectric polarization, the cubic anisotropy reflects the preferential orientation of the polarization along the $\langle 111\rangle$ axes and the isotropic random magnetic field corresponds to the randomly oriented local electric fields originating from the differing charges of the $\mathrm{Nb}^{5+}$ and $\mathrm{Zn}^{2+}$ $\left(\mathrm{Mg}^{2+}\right)$. Following a suggestion by Aharony $\underline{43}$, we consider the case where the Heisenberg term $H_{\text {Heisenberg }}$ dominates over the random field $H_{R F}$ which is larger than the contribution from the cubic anisotropy $H_{C u b i c}$ $\left(H_{\text {Heisenberg }}>H_{R F}>H_{C u b i c}\right) \underline{44}$

In this scenario there would be two distinct tempera- 
ture regions. At high temperatures the cubic anisotropy is irrelevant and therefore the system should behave like a Heisenberg model in a random field. In such a case the excitation spectrum is characterized by Goldstone modes and therefore no long-range order is expected in the presence of random fields. 45 The second temperature scale appears at low temperatures where the cubic anisotropy becomes relevant and therefore the system should be similar to that of an Ising system in the presence of a random field. In such a system we would expect long-range order to occur at equilibrium or for cooling in the presence of random fields, which typically yields a non-equilibrium state, local order with history dependent effects.

In the actual relaxor materials PMN and PZN, the Burns temperature $T_{d}$ marks the entry into the higher temperature region discussed above. Therefore, since the order parameter has a continuous symmetry ${ }^{40}$ and the excitation spectrum is characterized by gapless modes, the system does not order but forms polar nanoregions in a paraelectric background. On the other hand, the critical temperature $T_{c}$ characterizes the lower temperature region where the the system behaves more like an Ising model in the presence of a random field. This explains the local ferroelectric distortion characterized by the recovery of the soft-optic mode. Even though, in equilibrium, a 3D Ising system should have long-range order in the presence of small random fields, as found in magnetic systems, non-equilibrium effects with long time scales become dominant. The presence of non-equilibrium effects may explain the lack of true long-range order at low temperatures and the history dependence of physical properties like the linear birefringence. We also note that the presence of the phase-shifted polar nanoregions may also pose another energy barrier for the ordered phase at the critical temperature.

The low-temperature properties (below $\mathrm{T}_{c}$ ) of PZN and PMN are exactly analogous to that of $\mathrm{Mn}_{0.5} \mathrm{Zn}_{0.5} \mathrm{~F}_{2}$ in a magnetic field ${ }^{46.47}$ which is well described by a three- dimensional random field Ising model. In this system the bulk low temperature phase is characterized by nonequilibrium effects and therefore a disordered metastable state was observed. Importantly, an ordered phase was observed in the near-surface region of the sample, exactly analogous to the rhombohedral order observed in the near-surface region of PZN. The strong similarity between these two systems further affirms the model we have proposed. Our low-temperature phonon results are also qualitatively consistent with the observation of welldefined spin-waves in $\mathrm{Mn}_{0.5} \mathrm{Zn}_{0.5} \mathrm{~F}_{2}$ in the presence of random fields 48 A detailed, quantitative analysis of this model would be extremely valuable.

We have shown that PMN and PZN are very similar relaxor systems through identical behavior in the diffuse and phonon scattering. We do observe structural inhomogeneity at low temperatures in the PZN system in contrast to PMN. We speculate this difference is the result of stronger coupling in PZN between the ferroelectric order parameter and the unit cell shape. We suggest a consistent explanation for the relaxor transition in terms of the three-dimensional Heisenberg model with cubic anisotropy in the presence of random fields.

\section{Acknowledgments}

We thank A. Aharony for pointing out one of the key concepts introduced in this paper. We also are grateful to P. M. Gehring and G. Xu for useful discussions and M. M. Potter, J. J. -P. Bolduc, and L. E. McEwan for technical support. The work at the University of Toronto was supported by the Natural Science and Engineering Research Council of Canada. We acknowledge financial support from the U. S. DOE under contract No. DEAC02-98CH10886, and the Office of Naval Research under Grant No. N00014-99-1-0738.
1 S.-E. Park and T.R. Shrout, J. Appl. Phys. 82, 1804 (1997).

2 Z.-G. Ye, Key Engineering Materials Vols. 155-156, 81 (1998).

3 J. Kuwata, K. Uchino, and S. Nomura, Ferroelectrics 22, 863 (1979).

${ }^{4}$ G. Burns and F.H. Dacol, Solid Sate Commun. 48, 853 (1983).

5 A. Naberezhnov, S. Vakhrushev, B. Dorner, D. Strauch, and H. Moudden, Eur. Phys. J. B 11, 13 (1999).

6 P.M. Gehring, S. Wakimoto, Z.-G. Ye, and G. Shirane, Phys. Rev. Lett. 87, 277601 (2001).

7 P. M. Gehring, S.E. Park, G. Shirane, Phys. Rev. B 63, 224109 (2001).

${ }^{8}$ K. Hirota, Z.-G. Ye, S. Wakimoto, P.M. Gehring, and G. Shirane, Phys. Rev. B 65, 104105 (2002).

9 S. Wakimoto, C. Stock, Z.-G. Ye, W. Chen, P.M. Gehring, and G. Shirane, Phys. Rev. B 66, 224102 (2002).
10 Z.-G. Ye and H. Schmid, Ferroelectrics 145, 83 (1993).

11 S. Wakimoto, C. Stock, R.J. Birgeneau, Z.-G. Ye, W. Chen, W.J.L. Buyers, P.M. Gehring, and G. Shirane, Phys. Rev. B 65, 172105 (2002).

12 W. Cochran, Advan. Phys. 18, 157 (1969). W. Cochran, Advan. Phys. 9, 387 (1960).

13 S. Nomura, T. Takahashi, and Y. Yokomizo, J. Phys. Soc. Japan 27, 262 (1969).

14 A. Lebon, H. Dammak, G. Calvarin, and I.O. Ahmedou, J. Phys.: Condens. Matter 14, 7035 (2002).

15 D. La-Orauttapong, J. Toulouse, J.L. Robertson, and Z.G. Ye, Phys. Rev. B 64, 212101 (2001).

${ }^{16}$ G. Xu, Z. Zhong, Y. Bing, Z.-G. Ye, C. Stock, G. Shirane, Phys. Rev. B 67, 104102 (2003).

17 K. Ohwada, K. Hirota, P.W. Rehrig, Y. Fujii, and G. Shirane, Phys. Rev. B 67, 094111 (2003).

18 P.M. Gehring, W. Chen, Z.-G. Ye, and G. Shirane, unpublished (cond-mat/0304289). 
19 L. Zhang, M. Dong, and Z.-G. Ye, Mater. Sci. Eng. B 78, 96 (2000).

${ }^{20}$ We have estimated the domain size by fitting the Bragg peaks to the a lorentzian profile $1 /\left[1+(Q \xi)^{2}\right]$, where $\xi$, the correlation length, is used to estimate the domain size.

${ }^{21}$ M. Sakata, M.J. Cooper, K.D. Rouse, and B.T.M. Willis, Acta Cryst. A34, 336 (1978).

${ }^{22}$ H. You and Q.M. Zhang, Phys. Rev. Lett. 79, 3950 (1997).

23 T.Y. Koo, P.M. Gehring, G. Shirane, V. Kiryukhin, S.-G. Lee, and S.W. Cheong, Phys. Rev. B 65, 144113 (2002).

24 B. Dkhil, J.M. Kiat, G. Calvarin, G. Baldinozzi, S.B. Vakhrushev, and E. Suard, Phys. Rev. B 65, 024104 (2001).

25 J. Harada, J.D. Axe, and G. Shirane, Acta Cryst. A 26, 608 (1970).

26 G. Shirane, S.M. Shapiro, and J.M. Tranquada, Neutron Scattering with a Triple Axis Spectreometer (Cambridge University Press, Cambridge, 2002).

27 G. Shirane, J.D. Axe, J. Harada, and A. Linz, Phys. Rev. B 2, 3651 (1970).

28 J.D. Axe, J. Harada, and G. Shirane, Phys. Rev. B 1, 1227 (1970).

29 For a detailed discussion of the required substitutions see the appendices of R. A. Cowley, W. J. L. Buyers, P. Martel, R. W. H. Stevenson, J. Phys. C: Solid State Phys. 6, 2997 (1973).

30 G. Shirane, B.C. Frazer, V.J. Minkiewicz, J.A. Leake, and A. Linz, Phys. Rev. Lett. 19, 234 (1967).

31 A. Lebon, M.El Marssi, F. Farhi, H. Dammak, and G. Calvarin, J. Appl. Phys. 89, 3947 (2001).

32 I. Tomeno, S. Shimanuki, Y. Tsunoda, and Y. Ishii, J. Phys. Soc. Japan 70, 1444 (2001).

33 D. Viehland, S.J. Jang, L.E. Cross, and M. Wuttig, Phys. Rev. B 46, 8003 (1992).

34 B. Noheda, D.E. Cox, G. Shirane, S.E. Park, L.E. Cross,
Z. Zhong, Phys. Rev. Lett. 86, 3891 (2001)

35 B. Noheda, Z. Zhong, D.E. Cox, G. Shirane, S.E. Park, P. Rehrig, Phys. Rev. B 65, 224101 (2002).

36 B. Noheda, D.E. Cox, G. Shirane, Ferroelectrics 267, 147 (2002).

37 T. Iwase, H. Tazawa, K. Fujishiro, Y. Uesu, and Y. Yamada, J. Phys. Chem. Solid 60, 1419 (1999).

38 K. Fujishiro, T. Iwase, Y. Uesu, Y. Yamada, B. Dkhil, J.M. Kiat, S. Mori, and N. Yamamoto, J. Phys. Soc. Japan 69, 2331 (2000).

39 F. He, B.O. Wells, S.M. Shapiro, M.v. Zimmermann, A. Clark, and X.X. Xi, unpublished (cond-mat/0303317).

40 V. Westphal, W. Kleemann, and M.D. Glinchuk, Phys. Rev. Lett. 68, 847 (1992).

41 R. Pirc and R. Blinc, Phys. Rev. B 60, 13470 (1999).

42 R. Fisch, Phys. Rev. B 67, 094110 (2003).

43 A. Aharony, private communication.

44 We have considered another possible case where $H_{\text {Heisenberg }}>H_{C u b i c}>H_{R F}$. However in this case the physical properties should be similar to the case of an Ising model in the presence of random fields. Then there exists only one temperature scale where the zero-field cooled system, in equilibrium, is robust to random fields and a longrange ordered state is present at low temperatures. Thus, this may describe the physical properties around $\mathrm{T}_{c}$ but clearly does not predict the Burns temperature nor the polar nanoregions.

45 Y. Imry and S.K. Ma, Phys. Rev. Lett. 35, 1399 (1975).

46 J.P. Hill, T.R. Thurston, R.W. Erwin, M.J. Ramstad, and R.J. Birgeneau, Phys. Rev. Lett. 66, 3281 (1991).

47 J.P. Hill, Q. Feng, Q.J. Harris, R.J. Birgeneau, A.P. Ramirez, and A. Cassanho, Phys. Rev. B 55, 356 (1997).

48 R.L. Leheny, Y.S. Lee, G. Shirane, and R.J. Birgeneau, Eur. Phys. J. B 32, 287 (2003). 